\title{
Epipharyngeal Abrasive Therapy Down-regulates the Expression of SARS-CoV-2 Entry Factors ACE2 and TMPRSS2
}

\author{
KENSUKE NISHI $^{1,2}$, SHOHEI YOSHIMOTO ${ }^{3}$, SOICHIRO NISHI ${ }^{2}$, TOSHIYUKI TSUNODA ${ }^{4}$, \\ JUN OHNO $^{5}$, MICHINOBU YOSHIMURA ${ }^{6}$, KENJI HIROMATSU ${ }^{6}$ and TAKAFUMI YAMANO ${ }^{1}$ \\ ${ }^{1}$ Section of Otolaryngology, Department of Medicine, Fukuoka Dental College, Fukuoka, Japan; \\ ${ }^{2}$ Nishi Otolaryngology Clinic, Fukuoka, Japan; \\ ${ }^{3}$ Section of Pathology, Department of Morphological Biology, \\ Division of Biomedical Sciences, Fukuoka Dental College, Fukuoka, Japan; \\ ${ }^{4}$ Department of Cell Biology, Faculty of Medicine, Fukuoka University, Fukuoka, Japan; \\ ${ }^{5}$ Research Center for Regenerative Medicine, Fukuoka Dental College, Fukuoka, Japan; \\ ${ }^{6}$ Department of Microbiology and Immunology, Faculty of Medicine, Fukuoka University, Fukuoka, Japan
}

\begin{abstract}
Background: The epipharynx, with its high expression of Severe Acute Respiratory Syndrome Coronavirus 2 (SARS-CoV-2) entry factors angiotensinconverting enzyme 2 (ACE2) and transmembrane protease, serine 2 (TMPRSS2), is a primary target for SARS-CoV-2 replication in the early stage of Coronavirus Disease 19 (COVID-19). Epipharyngeal abrasive therapy (EAT) is a treatment for epipharyngitis in Japan which involves applying zinc chloride to the epipharyngeal mucosa. In this study, we evaluated the expression patterns of ACE2 and TMPRSS2 in tissue samples from patients before and after EAT. Patients and Methods: The study subjects were seven patients that had not been treated with EAT and 11 patients that had. For immunohistochemical assessment of the epipharyngeal mucosa, the staining intensity of ACE2 and TMPRSS2 was described as an immunohistochemical score (IHC score). Results: The IHC scores for ACE2 and TEMPRSS2 in the EAT-treated group were 3.40-fold and 1.81-fold lower, respectively, than those in the non-treated group ( $p=0.0208$ and $p=0.0244$, respectively). Conclusion: EAT down-regulates the expression of SARS-CoV-2 entry
\end{abstract}

This article is freely accessible online.

Correspondence to: Kensuke Nishi, MD, Ph.D., Section of Otolaryngology, Department of Medicine, Fukuoka Dental College, 2-15-1 Tamura, Sawara-ku, Fukuoka, 814-0193, Japan. Tel: +81 928010411, Fax: +81 928013678, e-mail: knishi@college.fdcnet.ac.jp

Key Words: Epipharyngeal abrasive therapy, EAT, SARS-CoV-2, ACE2, TMPRSS2. factors ACE2 and TMPRSS2. Thus, EAT has potential as a novel COVID-19 preventative method.

The epipharynx comprises ciliated epithelium and lymphoid tissue helped to prevent bacterial and viral infection; however, a recent study showed that severe acute respiratory syndrome coronavirus 2 (SARS-CoV-2), which causes COVID-19 disease, employs ciliary angiotensin-converting enzyme 2 (ACE2) and transmembrane protease, serine 2 (TMPRSS2) to enter and replicate within the epipharyngeal epithelium (1).

Epipharyngeal abrasive therapy (EAT) utilizes zinc chloride $\left(\mathrm{ZnCl}_{2}\right)$ for its astringent, denaturing, and antiinflammatory properties in the treatment of chronic epipharyngitis and related immune symptoms $(2,3)$. In addition, EAT is considered to be effective in preventing epipharyngitis caused by infectious pathogens, including viruses (4).

In this study, to examine whether EAT has potential as a method to prevent COVID-19, we evaluated the mRNA and protein expression patterns of SARS-CoV-2 entry factors ACE2 and TMPRSS2 in tissue samples from patients before and after EAT.

\section{Patients and Methods}

Patients and tissue samples. Patient information was obtained with permission from the Ethics Committee of Fukuoka Dental College (ID: 552). Samples were obtained by endoscopic epipharyngeal biopsy between July 2021 and August 2021. The subjects of this study were seven patients with chronic epipharyngitis that had not been treated with EAT (mean age $=53.3 \pm 18.3$ years) and 11 patients that had been treated with EAT for chronic epipharyngitis for 1 

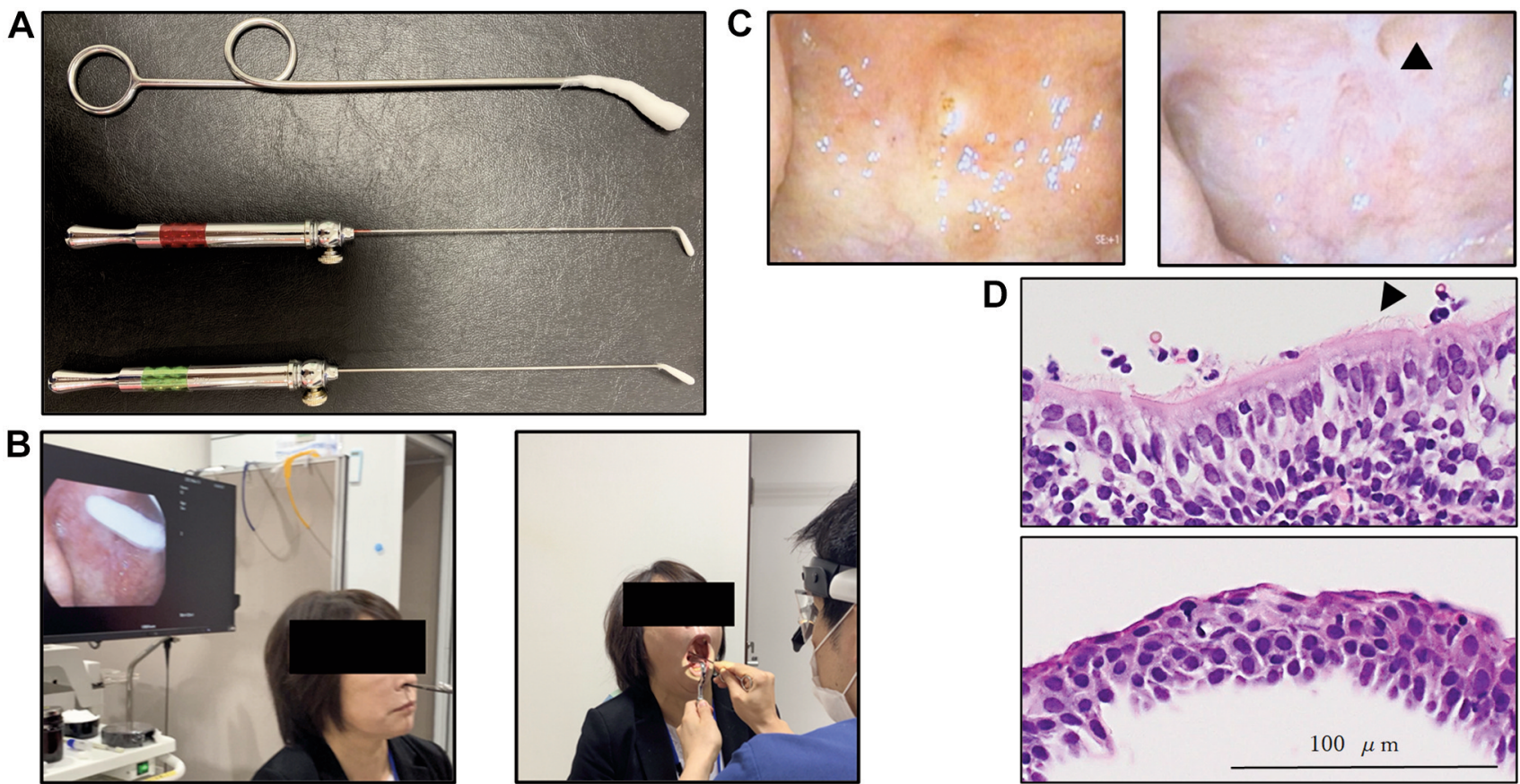

B
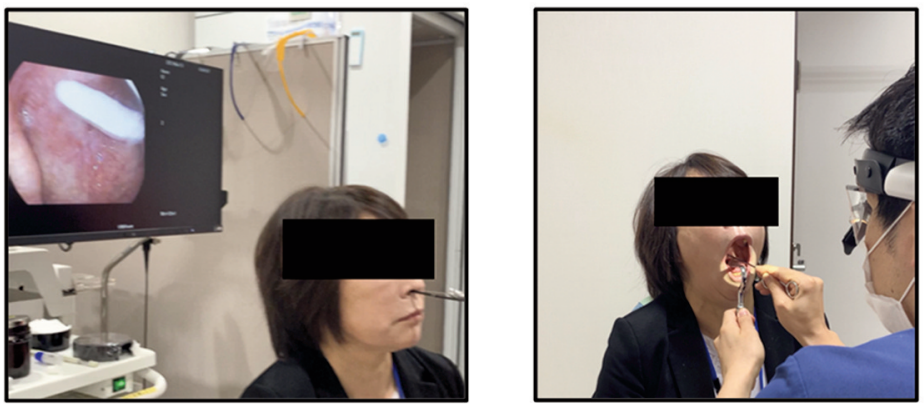

$100 \mu \mathrm{m}$

Figure 1. Epipharyngeal abrasive therapy (EAT). A: Cotton swabs for EAT. B: EAT procedure. Left panel shows the method of endoscopic EAT. Right panel shows the method of transoral EAT. C: Transnasal endoscopic photographs of the epipharynx in a patient. Left panel shows transnasal endoscopic photographs of the epipharynx pre-EAT treatment. Right panel shows transnasal endoscopic photographs of the epipharynx following 3 months of treatment with EAT. The arrowhead indicates the temporal whitening phenomenon. D: Hematoxylin and eosin (HE) staining of the epipharynx mucosa of a patient. Upper panel shows HE staining of the epipharynx pre-EAT treatment. Lower panel shows HE staining of the epipharynx following 3 months of EAT treatment. The arrowhead indicates cilia on the surface of epithelial cells.

month or more (mean age $=64.4 \pm 11.9$ years). All participants provided informed written consent. The research was conducted in accordance with the Declaration of Helsinki and Title 45, US Code of Federal Regulations, Part 46, Protection of Human Subjects, effective as of December 13, 2001.

EAT. Zinc chloride (1\%) was applied to the epipharyngeal mucosa of patients in the Outpatient Department one to three times a week by a Board-certified otorhinolaryngologist using a cotton swab (Figure 1A and B), as described previously $(2,3)$.

Antibodies. Primary rabbit antibody to ACE2 (HPA000288) was purchased from Sigma-Aldrich (St. Louis, MO, USA) and rabbit antibody to TMPRSS2 (\#14437-1-AP) was purchased from PROTEINTECH (Rosemont, IL, USA) The secondary horseradish peroxidase (HRP)-conjugated polymer anti-rabbit antibody was purchased from DAKO-Agilent Technologies Co. (Santa Clara, CA, USA).

Immunohistochemical (IHC) staining and RNA in situ hybridization. Neutral buffered formalin (10\%)-fixed and paraffin-embedded tissue blocks were cut into $4 \mu \mathrm{m}$-thick sections for hematoxylin-eosin and IHC staining. Antigen retrieval was performed for all sections by an autoclave treatment at $121^{\circ} \mathrm{C}$ for 5 min in $0.01 \mathrm{M}$ citrate buffer, $\mathrm{pH}$ 6.0. Immunostaining was performed by using EnVision/HRP kit (DAKO). Briefly, the sections were treated with a $0.1 \%$ hydrogen peroxide-methanol solution to inhibit endogenous peroxidase activity and a $5 \%$ bovine serum albumin/tris buffered saline to block any non-specific binding of primary antibodies. Subsequently, each section was incubated with the primary antibody against ACE2 (1:100 dilution) or TMPRSS2 (1:100 dilution) at $4^{\circ} \mathrm{C}$ overnight. These sections were then incubated with HRP-conjugated polymer anti-rabbit or anti-mouse antibody. The peroxidase activity was visualized using $0.1 \%$ 3,3'-diaminobenzidine and $0.01 \%$ hydrogen peroxide in tris buffered saline. for ihc assessment, the staining intensity of slides was described as an IHC score (scored on a scale of $0-3 ; 0$ : negative, 1 : weakly positive, 2 : intermediately positive, 3: strongly positive). To detect ACE2/TMPRSS 2 mRNA on the tissue, the in situ hybridization system RNA scope (Advanced Cell Diagnostics, Hayward, CA, USA) was used following the manufacturer's guidelines. The expression levels of mRNA were qualitatively evaluated.

Statistical analysis. All data are expressed as the mean \pm standard error of the mean. Mann-Whitney $U$-tests were applied for comparisons between two groups. Statistical significance was set as $p<0.05$.

\section{Results}

Continuous EAT resulted in reduced redness and temporary whitening of the epipharyngeal mucosa, which was considered to indicate reduced inflammation (Figure 1C). In 
the group not treated with EAT, the epipharyngeal mucosa comprised ciliated epithelium, whereas in the EAT-treated group, squamous metaplasia was evident in the epipharyngeal mucosa (Figure 1D). Importantly, ACE2/TMPRESS2 expression was not detected in the region of squamous metaplasia in the EAT-treated group, as determined by IHC and mRNA in situ hybridization (Figure 2A). The IHC scores for ACE2 and TEMPRSS2 in the EAT-treated group were 3.40-fold and 1.81-fold lower than those in the non-treated group, respectively (ACE2: $p=0.0208$, TMPRSS2: $p=0.0244$; Figure 2B).

\section{Discussion}

EAT was originally developed in Japan as a treatment to reduce chronic epipharyngitis, which causes various upper respiratory tract symptoms such as postnasal drip, throat pain and cough (3). In addition, EAT has been reported to be effective in improving symptoms of autoimmune diseases such as IgA nephropathy and psoriatic arthritis associated with chronic epipharyngitis. (2, 5, 6). A former study showed that continuous EAT macroscopically induces mucosal whitening, defined as temporal whitening phenomenon (7), suggesting that $\mathrm{ZnCl}_{2}$ utilized in EAT induces an astringent effect on inflamed mucosa (2). We microscopically found that EAT induces squamous metaplasia, resulting in the disappearance of ciliary structures exhibiting expression of SARS-CoV-2 entry factors ACE2 and TMPRSS2. Thereby, the mRNA expression of ACE2 and TMPRSS2 was reduced. These results suggested that EAT transcriptionally regulates expression of ACE2 and TMPRSS2 during squamous metaplasia, where these proteins were previously suggested to be absent (1). SARS-CoV-2 enters human cells via binding to ACE2, followed by its priming by TMPRSS2 (8). Therefore, these proteins are potential targets for antiviral intervention for the prevention of SARS-CoV-2 infection or in related treatment (9). In one study, TMPRSS2 inhibitor was not found to be effective at preventing SARS-CoV-2 infection (10), suggesting that targeting TMPRSS2 alone is not critical for preventing viral infection. In contrast, recent studies indicate that nasal administration of ACE2 inhibitor or vaccine is passably effective for SARS-CoV-2 prevention $(1,11)$. However, these approaches require further investigation and dual inhibition is suggested to be more critical for preventing viral infection. EAT is a simple method of reducing ciliary ACE2/TMPRSS2 expression. No intervention study has been conducted to investigate the preventative effect of EAT on SARS-CoV-2 infection. However, as lower ACE2 and TMPRSS2 expression might be helpful in reducing acquisition of SARS-CoV-2 infection in the upper airway $(12,13)$, EAT may have potential as a novel method for preventing COVID-19.
A
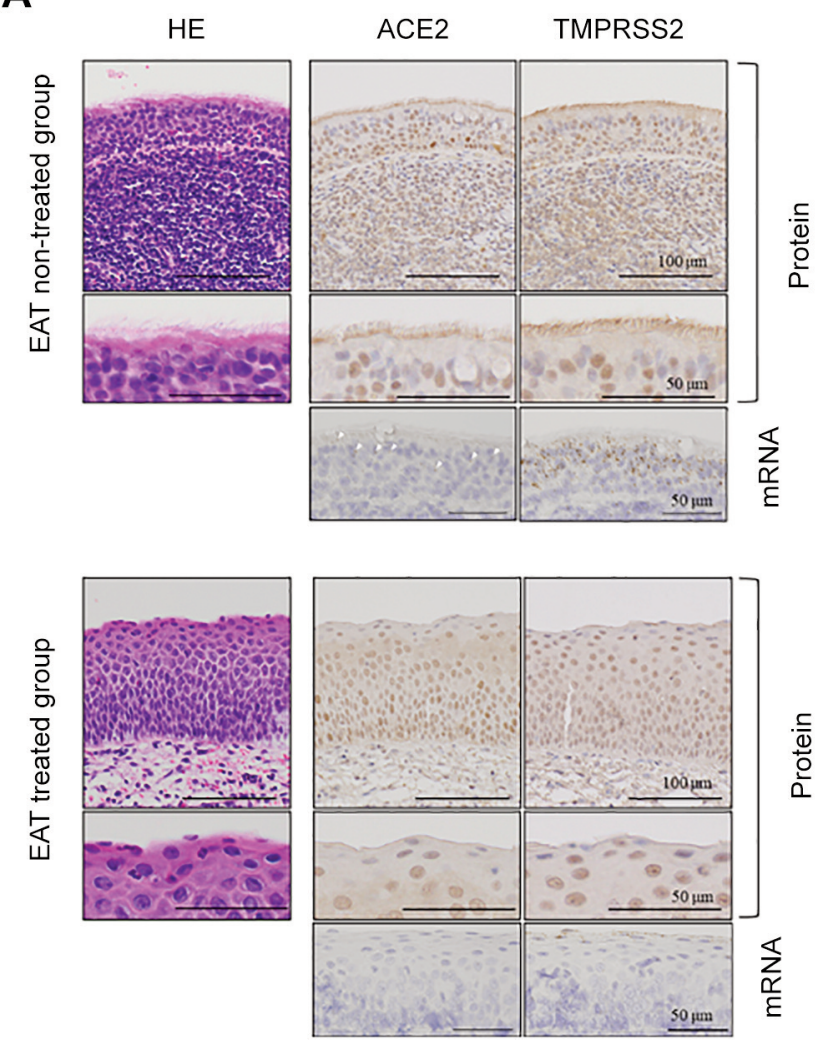

B
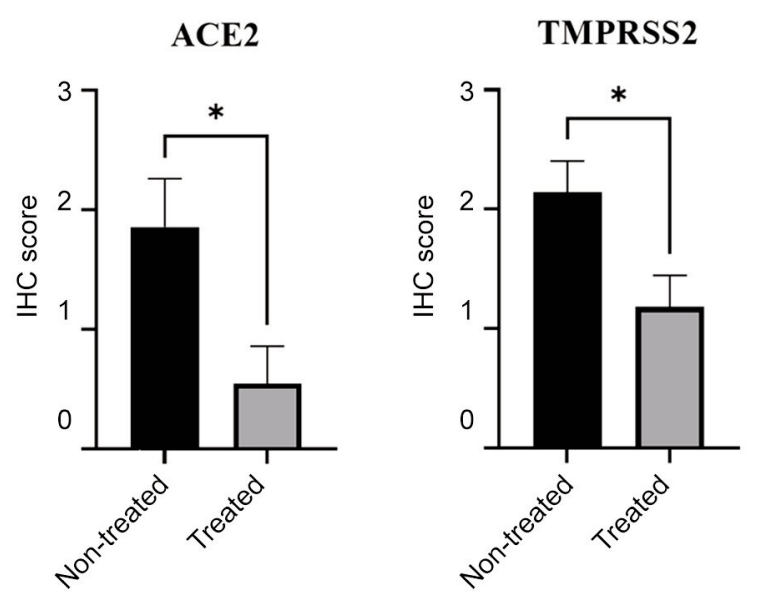

Figure 2. $m R N A$ and protein expression patterns of angiotensinconverting enzyme 2 (ACE2) and transmembrane protease, serine 2 (TMPRSS2) in patient tissue samples without and with epipharyngeal abrasive therapy (EAT). A: ACE2 and TMPRSS2 expression in the epipharynx of the EAT-treated and non-treated groups. The upper and middle panels show the protein expression patterns. The lower panels show the mRNA expression patterns. Arrowheads indicate the mRNA expression of ACE2. B: Immunohistochemical (IHC) scores for ACE2 and TMPRSS 2 expression on the epipharyngeal mucosa of the EATtreated $(n=11)$ and non-treated groups $(n=7)$. *Significantly different at $p<0.05$. 


\section{Conflicts of Interest}

The Authors declare that they have no competing interests.

\section{Authors' Contributions}

K.N. and S.Y. designed the study, performed the analyses, analyzed the data and wrote the article; J.O. and T.Y. collected the data; S.N. gave technical supports; T.T., M.Y., and K.H. gave conceptual advice.

\section{Acknowledgements}

The Authors thank Dr. Hiroaki Tanaka for his technical assistance regarding EAT.

\section{References}

1 Sungnak W, Huang N, Bécavin C, Berg M, Queen R, Litvinukova M, Talavera-López C, Maatz H, Reichart D, Sampaziotis F, Worlock KB, Yoshida M, Barnes JL and HCA Lung Biological Network: SARS-CoV-2 entry factors are highly expressed in nasal epithelial cells together with innate immune genes. Nat Med 26(5): 681-687, 2020. PMID: 32327758. DOI: 10.1038/s41591-020-0868-6

2 Hotta O, Inoue C, Tanaka A and Ieiri N: Possible mechanisms underlying epipharyngeal abrasive therapy (EAT) with $\mathrm{ZNCl} 2$ solution for the treatment of autoimmune diseases and functional somatic syndrome. J Antivir Antiretrovir 9(4): 81-86, 2017. DOI: 10.4172/1948-5964.1000168

3 Mogitate M, Sasaki Y and Komiyama A: Outcome of an outpatient specialty clinic for chronic epipharyngitis. Auris Nasus Larynx 48(3): 451-456, 2021. PMID: 33067050. DOI: 10.1016/j.anl.2020.09.019

4 Hotta O, Tanaka A, Torigoe A, Imai K, Ieiri N and Japanese Focal Inflammation Research Group: Involvement of chronic epipharyngitis in autoimmune (auto-inflammatory) syndrome induced by adjuvants (ASIA). Immunol Res 65(1): 66-71, 2017. PMID: 27592233. DOI: 10.1007/s12026-016-8859-x

5 Kaneko T, Mii A, Fukui M, Nagahama K, Shimizu A and Tsuruoka S: IgA nephropathy and psoriatic arthritis that improved with steroid pulse therapy and mizoribine in combination with treatment for chronic tonsillitis and epipharyngitis. Intern Med 54(9): 1085-1090, 2015. PMID: 25948353. DOI: 10.2169/internalmedicine.54.3510

6 Fujimoto M, Katayama K, Nishikawa K, Mizoguchi S, Oda K, Hirabayashi Y, Suzuki Y, Haruki A, Ito T, Murata T, Ishikawa E, Sugimura $\mathrm{Y}$ and Ito $\mathrm{M}$ : A kidney transplant recipient with recurrent Henoch-Schönlein purpura nephritis successfully treated with steroid pulse therapy and epipharyngeal abrasive therapy. Nephron 144 Suppl 1: 54-58, 2020. PMID: 33221802. DOI: $10.1159 / 000511166$
7 Tanaka A: Studies on band-limited light endoscopic diagnosis and endoscopic epipharyngeal abrasive therapy in chronic epipharyngitis. Stomato-pharyngeal 31(1): 57-67, 2018. DOI: 10.14821/stomatopharyngology.31.57

8 Lukassen S, Chua RL, Trefzer T, Kahn NC, Schneider MA, Muley T, Winter H, Meister M, Veith C, Boots AW, Hennig BP, Kreuter M, Conrad C and Eils R: SARS-CoV-2 receptor ACE2 and TMPRSS2 are primarily expressed in bronchial transient secretory cells. EMBO J 39(10): e105114, 2020. PMID: 32246845. DOI: 10.15252/embj.20105114

9 Wu CY, Lin YS, Yang YH, Shu LH, Cheng YC and Liu HT: Potential simultaneous inhibitors of angiotensin-converting enzyme 2 and transmembrane protease, serine 2. Front Pharmacol 11: 584158, 2020. PMID: 33390952. DOI: 10.3389/fphar.2020.584158

10 Gunst JD, Staerke NB, Pahus MH, Kristensen LH, Bodilsen J, Lohse N, Dalgaard LS, Brønnum D, Fröbert O, Hønge B, Johansen IS, Monrad I, Erikstrup C, Rosendal R, Vilstrup E, Mariager T, Bove DG, Offersen R, Shakar S, Cajander S, Jørgensen NP, Sritharan SS, Breining P, Jespersen S, Mortensen KL, Jensen ML, Kolte L, Frattari GS, Larsen CS, Storgaard M, Nielsen LP, Tolstrup M, Sædder EA, Østergaard LJ, Ngo HTT, Jensen MH, Højen JF, Kjolby M and Søgaard OS: Efficacy of the TMPRSS2 inhibitor camostat mesilate in patients hospitalized with Covid-19-a double-blind randomized controlled trial. EClinicalMedicine 35: 100849, 2021. PMID: 33903855. DOI: 10.1016/j.eclinm.2021.100849

11 Zou Z, Yan Y, Shu Y, Gao R, Sun Y, Li X, Ju X, Liang Z, Liu Q, Zhao Y, Guo F, Bai T, Han Z, Zhu J, Zhou H, Huang F, Li C, Lu H, Li N, Li D, Jin N, Penninger JM and Jiang C: Angiotensin-converting enzyme 2 protects from lethal avian influenza A H5N1 infections. Nat Commun 5: 3594, 2014. PMID: 24800825. DOI: $10.1038 /$ ncomms 4594

12 Patel AB and Verma A: Nasal ACE2 levels and COVID-19 in children. JAMA 323(23): 2386-2387, 2020. PMID: 32432681. DOI: $10.1001 /$ jama.2020.8946

13 Hasan MR, Ahmad MN, Dargham SR, Zayed H, Al Hashemi A, Ngwabi N, Perez Lopez A, Dobson S, Abu Raddad LJ and Tang P: Nasopharyngeal expression of angiotensin-converting enzyme 2 and transmembrane serine protease 2 in children within SARSCoV-2-infected family clusters. Microbiol Spectr: e0078321, 2021. PMID: 34730438. DOI: 10.1128/Spectrum.00783-21

Received October 31, 2021

Revised November 29, 2021

Accepted November 30, 2021 